\title{
Abstención electoral y gobiernos progresistas en Latinoamérica: Nicaragua y Venezuela
}

\author{
Wilson René González-Cortés*
}

Candidato a Maestría en Estudios Políticos Latinoamericanos. Docente investigador, Facultad de Derecho, Universidad Cooperativa de Colombia, Bucaramanga, Colombia.

Correo electrónico: wgonzalez@campusucc.edu.co

Recibido: 22 de agosto del 2014 Aprobado: 29 de septiembre del 2014

Cómo citar este artículo:

Wilson René González-Cortés. Abstención electoral y gobiernos progresistas en Latinoamérica: Nicaragua y Venezuela. IIx। 20. Diciembre 2014. Pág. 77. doi: http://dx.doi. org/10.16925/di.v16i20.836

\section{Resumen}

El presente artículo tiene como objetivo presentar los cambios y continuidades en la abstención electoral, a partir de la irrupción de gobiernos con tendencias izquierdistas en América Latina. Desde comienzos de la década de los noventa del siglo pasado, han sido elegidos en América Latina una serie de gobiernos de carácter alternativo a las políticas tradicionales, dentro de los cuales Nicaragua y Venezuela son los únicos que conservan el sufragio como un derecho y no como un deber u obligación. Consideramos que las elecciones son un mecanismo que permite verificar el nivel de legitimidad de estos gobiernos, así como los índices de abstención permiten cotejar en qué medida se crean democracias más estables bajo esas nuevas políticas. Desde una mirada general al papel de la legitimidad en el sistema político, señalaremos el papel de las elecciones en los sistemas democráticos de orden liberal burgués. Veremos el carácter de los gobiernos denominados alternativos y examinaremos la evolución del fenómeno abstencionista en los dos países señalados. Las cifras de abstención electoral en estos dos países, desde comienzos de la década de los años ochenta del siglo xx, muestra diversas inclinaciones que bien vale la pena considerar frente a la caracterización de los respectivos gobiernos.

Palabras clave: abstencionismo, alternativo, elecciones, legitimidad.

\section{Electoral Abstention and Progressive Governments in Latin America: Nicaragua ANd Venezuela}

\begin{abstract}
This article aims to present the changes and continuities in electoral abstention, starting from the eruption of governments with leftist tendencies in Latin America. Since the early 1990s, a series of governments have been elected in Latin America as alternatives to traditional politics, among which only Nicaragua and Venezuela conserve voting as a right rather than a duty or obligation. We believe that elections are a mechanism that enables verification of the level of legitimacy of these governments, so that abstention levels make it possible to determine to what extent those new policies foster the creation of more stable democracies. Taking a general approach to the role of legitimacy in the political system, we will look at the role of elections in bourgeois liberal democratic systems. We will examine the character of so-called alternative governments along with the evolution of the abstentionist phenomenon in the two abovementioned countries. Their electoral abstention figures since the early 1980s show diverse trends worthy of consideration when characterizing their respective governments.
\end{abstract}

Keywords: abstentionism, alternative, elections, legitimacy.

\section{Abstenção eleitoral e governos progressistas na América Latina: Nicarágua e Venezuela}

\section{Resumo}

El presente artigo tiene como objetivo presentar los cambios y continuidades na abstenção electoral, a partir da irrupção de gobiernos con tendencias izquierdistas en América Latina. Desde comienzos da década dos años noventa del siglo pasado han sido elegidos en América Latina una serie de gobiernos de carácter alternativo a las políticas tradicionales, dentro dos cuales Nicaragua y Venezuela son los únicos que conservan el sufragio como un direito y no un deber u obligação. Consideramos que las eleccoes son un mecanismo que permite verificar el nivel de legitimidad de estos gobiernos, así como los índices de abstenção permiten cotejar en qué medida se crean democracias más estables bajo esas nuevas políticas. Desde una mirada general al papel da legitimidad no sistema político, señalaremos el papel das eleccoes en los sistemas democráticos de orden liberal burgués. Veremos el carácter dos gobiernos denominados alternativos y examinaremos la evolução del fenómeno abstencionista en los dos países señalados. Las cifras de abstenção electoral en estos dos países desde comienzos da década dos años ochenta del siglo xx, muestra diversas inclinacoes que bien vale la pena considerar frente a la caracterização dos respectivos gobiernos.

Palavras-chave: abstencionismo, alternativo, eleccoes, legitimidad. 


\section{EL PAPEL DE LA LEGITIMIDAD EN EL SISTEMA POLÍTICO}

La abstención electoral ha sido considerada como una forma de medir en las urnas la legitimidad de un régimen político. La legitimidad en los gobiernos es un postulado esencial que conlleva dos elementos: el principio de soberanía colectiva en el pueblo o en la nación y una técnica de elección que se basa en criterios de mayorías. Bien sea mediante elecciones o por el ostensible apoyo revolucionario, el papel del pueblo en la conformación o mantenimiento del poder ha pasado a convertirse en un axioma de la política a partir de la modernidad.

Maquiavelo, con su aguda racionalidad, sostenía ante su príncipe la importancia de llegar al poder con el apoyo del pueblo: "El que llega [al principado] por el favor popular es única autoridad, y no tiene en derredor a nadie o casi nadie que no esté dispuesto a obedecer". ${ }^{1}$

Ningún poder que se pretenda identificar como democrático puede sostenerse exclusivamente en la fuerza, requiere un mínimo de consenso que haga posible la denominada "gobernanza". Según Weber, ninguna dominación se contenta con tener como posibilidades de persistencia motivos exclusivamente de orden material, afectivos o racionales con arreglo a valores, sino que todo tipo de dominación procura fomentar la creencia de su "legitimidad".

La viabilidad de las elecciones como mecanismo de decisión no es un patrimonio exclusivo de Occidente, ni de los regímenes políticos demoliberales. La democracia radical (ya propuesta por Rousseau), que se buscó materializar en los sistemas de asamblea existentes en los países socialistas durante el siglo $\mathrm{xx},{ }^{3}$ así como en la denominada democracia participativa de los Estados que se proclaman sociales y de derecho, junto con el respeto del acto electoral representativo que acoge la fórmula de Sieyes en la mayoría de países del mundo, son el complejo de posibilidades que otorgan hoy a la elección una técnica democrática para configurar el consenso.

1. Nicolás Maquiavelo. El PrínCipe. Editorial Porrúa. (1994). Pág. 16.

2. Max Weber. Economía y sociedad. Fondo de Cultura Económica. (1997). Pág. 170.

3. La Constitución de la URSS de 1918 establecía en el artículo 64 el derecho de sufragio activo y pasivo, esto es, el derecho a elegir o a ser elegido para los Soviets, que fueron los órganos que detentaron el poder central y local. De igual manera, la Constitución de 1936 de la URSS estableció a partir del artículo 134 el sistema electoral, garantizando un derecho al sufragio universal, igual, directo y secreto, a partir de los dieciocho años.
La mayoría como legitimidad se complementa con un procedimiento electoral para identificar la democracia. Que la mayoría elija se asocia con la idea de la mayoría manda, así como con la idea de la mayoría no se equivoca. Es la condena a la excepción como opción racional, pero en la mente de esa mayoría persiste la convicción de que se libera la culpa cuando muchos se equivocan. Es claro que estas construcciones lógicas pueden ser objeto de variadas observaciones, que van desde el analfabetismo político de la mayoría, hasta la ya extendida denuncia: los candidatos siempre sirven a una minoría no obstante ser elegidos por la mayoría.

Pero la mayoría en la historia tuvo otro propósito. Una legitimidad definida en términos de mayoría se impone como ruptura con un mundo antiguo donde las minorías dictaban su ley. Para Rosanvallon, en la elección democrática se mezclan unas ficciones fundadoras, el principio de justificación y una técnica de decisión. ${ }^{4}$ La primera es la concreción de un sujeto político que bien puede ser el pueblo o la nación. El elegido representa a la colectividad, quien detenta la soberanía como ruptura de los regímenes autocráticos. Somos la democracia es lo que se busca proclamar. Mediante la segunda (la democracia como técnica de decisión), se trata de un procedimiento práctico de elección. Busca configurarse una máquina cuyo proceso va de un sistema de entrada de información o input (que se materializa en el acto de ejercer el derecho al sufragio), a un output como producto de las diversas voluntades, la designación del elegido. Esta máquina bien puede ser denominada como el sistema electoral.

Estamos de acuerdo con la primera de ellas: es el pueblo la fuente del poder democrático. Sin embargo, es necesario cuestionarse sobre la validez de las elecciones como mecanismo idóneo para garantizar que un gobierno esté al servicio de la voluntad general. Es cuestionable especialmente en sociedades altamente fragmentadas - como en el caso del tercer mundo- que exista esa voluntad común de todo el conglomerado social. Esta es una idea liberal que desconoce la existencia de múltiples intereses en la sociedad. Asimismo, puede cuestionarse el método de la elección basada en las mayorías como expresión de la voluntad general, ya que limita la expresión real de las ideas de las minorías. En los métodos de elección por mayorías, quienes no hacen parte de ellas siempre verán su expresión como parte del ideario contrahegemónico en el sentido gramsciano de

4. Pierre Rosanvallon. LA LegitimidAD DEMOCRÁtiCA: IMPARCIALIDAD, REFLEXIVIDAD y PRoXimidad. Ediciones Paidós Ibérica. (2010). Pág. 22. 
la expresión, esto es, como una forma en que el grupo social subordinado o minoritario construye y mantiene su propia identidad, que aspira a ser dominante y asimilado como el adecuado por la mayoría. Una réplica más al método de las mayorías puede extraerse del propio desarrollo histórico de la ciencia y la cultura: a menudo la mayoría se equivoca y una minoría es poseedora de las ideas que habrán de ser las ideas dominantes en un futuro, como ocurrió con la teoría cosmológica o varias de las leyes científicas.

\section{DEMOCRACIA Y ABSTENCIÓN ELECTORAL}

$\mathrm{Al}$ ser las elecciones un desarrollo de la legitimidad democrática, dentro de la ideología dominante, la no participación en ellas puede interpretarse como una medida antidemocrática. Al ser la democracia electoral la forma como se materializa la soberanía del colectivo, la no participación en las elecciones puede entenderse como una forma elitista de asumir la política. Claro que esto puede entenderse si se entiende el sufragio como un deber, pero no como un derecho de carácter político y plenamente renunciable.

La abstención electoral es la renuncia consciente o inconsciente a ese derecho. El concepto de abstención electoral como expresión de implicaciones y contenido político es objeto de ásperas polémicas. La abstención electoral ha sido considerada como la no participación en el acto de emitir el sufragio teniendo derecho a él, es la renuncia consciente o inconsciente a un derecho, la cual, de acuerdo con el Instituto Interamericano de Derechos Humanos, "se enmarca en el fenómeno más amplio de la apatía participativa, es un indicador de la participación: muestra el porcentaje de los no votantes sobre el total de los que tienen derecho de voto". ${ }^{5}$

Para determinar la abstención, es entonces previamente necesario saber quiénes tienen derecho al voto, lo cual se precisa a partir del denominado censo electoral. Acto seguido, se realiza una operación aritmética, restando del censo electoral el número de participantes en las elecciones, para tomar así por resultado el porcentaje de abstención. Algunos autores señalan que el significado de la abstención electoral comprende unos

\footnotetext{
5. Instituto Interamericano de Derechos Humanos y Centro Interamericano de Asesoría y Promoción Electoral. Diccionario electoral. Tomo I. (2000). Pág. 1. [De aquí en adelante IIDH y CAPEL].
}

elementos que la hacen más amplia (en lo que denominan abstención activa, la cual se trata además de la generalmente aceptada no concurrencia a las urnas), de aquellas opciones que se separan de la "oferta" electoral partidaria, como el caso del voto en blanco y el voto anulado de manera intencional. ${ }^{6}$ Ahora bien, como lo señala un importante sector de la doctrina, "quien concurre a las urnas y emite un voto en blanco tiene más elementos en común -al menos objetivamente hablando- con quien vota por un partido determinado que con quien no concurre al recinto de votación".

Si se tiene en cuenta la disputada historia por la consagración del sufragio universal como derecho, es válido preguntarse ¿por qué habría alguien de renunciar a tan preciada oportunidad de decidir sobre el ejercicio del poder? Las respuestas pueden variar por distintos aspectos, desde lo intuitivo hasta lo científico, pero la más generalizada o común apunta a la denominada apatía política.

En este punto, es preciso tener en cuenta que no se vota por razones diversas. Tres grandes tipos de causas se señalan como fundamento para la abstención: motivos técnicos, desinterés o apatía, y motivos políticos. ${ }^{8}$ Sin embargo, aquí es preciso señalar que la denominada apatía no corresponde al sentido que a esta daban los estoicos en la antigüedad, como ese estado de condición ideal para meditar, de imperturbabilidad anímica en que los sentimientos no son obstáculo a la actividad de la razón. ${ }^{9}$ Se trata más bien de una falta de importancia o desinterés hacia a la situación política electoral que pareciera generada por el desencanto o la frustración.

El abstencionismo es entonces una tendencia sistemática hacia la abstención que, según Raventos, es problemática cuando tiende a concentrarse en sectores específicos de la población, o cuando es un indicio de malestar con la política y la conducción de los asuntos públicos. ${ }^{10}$ Tres escenarios se suelen identificar como respuesta de los gobiernos a la abstención electoral: el voto

6. Cf. Juan Hernández. Abstención activa. Diccionario CRítico DE CIENCIAs SOCIALES. Universidad de La Laguna. (2004).

7. José Thompson. Abstencionismo y participación electoral. Tratado De derecho electoral comparado de América Latina. Fondo de Cultura Económica. (2007). Pág. 267.

8. Ciska Raventós. Abstención: Conceptos y AlCANCES, PRoblemas Asociados y PosibILIDAdes De SUPERACIÓN. PERSPECTIVA POLÍticA. XVII Conferencia Protocolo de Tikal. Centro Interamericano de Asesoría y Promoción Electoral (CAPEL). (2003).

9. Cf. Mark Moisevich Rosental y Pavel Fedorovich Iudin. Diccionario soviético DE FILOsofía. Ediciones Pueblos Unidos. (1965).

10. Véase Ciska Raventós, supra, nota 8. 
libre, como en los casos de Nicaragua y Venezuela, esto es, que simplemente se consagra el derecho al sufragio sin imposiciones ni limitaciones. Lo señala Aragón:

Ahora bien, el sufragio en libertad no significa sólo que el acto de votar deba hacerse sin coacción alguna y con plena capacidad de opción (votar sí o no si se trata de un referéndum, o a una u otras candidaturas si se trata de elecciones, o en blanco en cualquier caso, o incluso no votar, si se prefiere), sino que el propio derecho de sufragio ha de estar acompañado de otras libertades sin las cuales no podría hablarse con propiedad de un sufragio libre, como las libertades de expresión, asociación, reunión y manifestación, y por supuesto la libertad de presentación de candidaturas (con las modulaciones necesarias para dotar de eficacia al acto electoral), acompañada de los principios de libre concurrencia entre ellas y de libre desarrollo de la campaña electoral. ${ }^{11}$

De esta forma, todo tipo de incentivo o limitación será una restricción a la libertad del sufragio.

Otro de los mecanismos que se ha utilizado es el voto obligatorio. Este tipo de voto ha sido el más frecuente mecanismo en América Latina para conjurar la abstención electoral. Pero vale la pena preguntarse ¿qué tipo de democracia es esa que tiene que utilizar la sanción para que la gente concurra a las urnas? No obstante, varios países del continente tienen el sufragio como una obligación, y se impone una sanción a quienes no participen en las elecciones, tal como sucede en países como Argentina, Bolivia, Brasil, Chile, Costa Rica, Ecuador, El Salvador, Honduras, Paraguay, Perú y Uruguay. La tercera opción es la existencia del derecho al sufragio como un deber, pero sin sanción en caso de incumplimiento, como en los casos de Colombia, Guatemala, México y Panamá.

\section{Gobiernos ALTERNATIVOS EN AMÉRICA LATINA ${ }^{12}$}

La dinámica política latinoamericana ha evidenciado desde comienzos de los años noventa del siglo $\mathrm{xx}$, la

11. Manuel Aragón. Derecho de sufragio: principio y función. Tratado de derecho electoral comparado de América latina. Fondo de Cultura Económica. (2007)

12. El presente capítulo ha sido redactado a partir de nuestro artículo anterior sobre abstencionismo y las elecciones presidenciales en Venezuela, próximo a ser publicado por la Universidad Cooperativa de Colombia, Medellín. irrupción de nuevas formas políticas. La transición en Chile de la dictadura de Pinochet hacia el primero de los gobiernos de concertación para la democracia que encabezara Patricio Alwin, así como otras situaciones del escenario mundial tales como la Perestroika, iniciaron una era de cambios gubernamentales en varios países de América Latina, propiciando un giro hacia la centroizquierda o los gobiernos alternativos, y creando nuevos escenarios políticos de cara a la tradicional relación con los centros de poder mundial. ${ }^{13}$

Sobre el concepto de izquierda o alternativos con que se denominan algunos gobiernos que irrumpieron en el panorama latinoamericano, se encuentra una polémica que se bifurca en la discusión sobre qué es izquierda o qué es verdaderamente alternativo, pero existe consenso sobre la temporalidad y espacialidad de estos conceptos, así como sobre su sentido de mutación acentuada. Lo alternativo bien puede definirse - como lo recoge el Profesor Alejo Vargas- en la dinámica política electoral latinoamericana:

Usamos la expresión alternativos para referirnos a gobiernos - nacionales o locales- que no proceden de partidos políticos tradicionales, sino más bien de formaciones o coaliciones novedosas, que pueden o no reivindicarse de la izquierda tradicional, pero que, en general, sí incorporan fuerzas de esa procedencia y cuyas políticas tienen algunos rasgos similares: búsqueda de mayor autonomía frente a la de los EE.UU., énfasis en lo social dentro de su política pública, búsqueda de sintonías con las demandas de los sectores sociales mayoritarios, búsqueda de nuevas relaciones civiles-militares. ${ }^{14}$

El fenómeno de los gobiernos alternativos en América Latina no es nuevo, pero solía darse desde plataformas más aisladas y desde un reducido caudillismo que rememora distintos lugares y tiempos: Getulio Vargas en Brasil, José María Velasco en Ecuador, Lázaro Cárdenas del Río en México, Jacobo Arbenz en Guatemala, Juan Domingo Perón en Argentina y Rómulo Betancourt en Venezuela, sin contar desde luego con movimientos más orgánicos como la Revolución cubana o el ascenso de la Unidad Popular con Allende en Chile, o el triunfo del movimiento sandinista en Nicaragua. Hoy se habla de una corriente histórica en que

13. Beatriz Stolowicz. Gobiernos de izQuierda en América Latina. Ediciones Aurora. (2008).

14. Alejo Vargas. Viejas y nuevas izquierdas y los gobiernos alternativos en América Latina. Coord. Jairo Estrada. MARX vive: IZqUIERdA y socialismo en América latina. (2008). Pág. 125. 
la excepcionalidad ha cambiado y que vincula a varias de las repúblicas presidencialistas latinoamericanas. ${ }^{15}$

Desde la transición en 1990 de la dictadura de Pinochet a Patricio Alwin, siendo el primero de los cuatro gobiernos de la Concertación de Partidos por la Democracia, son varios los cambios gubernamentales que se han sucedido en América Latina.

La Unión Cívica Radical y el Partido Justicialista se han disputado como acuerdos de fuerzas de centroizquierda, lo propio con el Gobierno argentino, aunque no pueden incluirse dentro de este movimiento todos los gobiernos del Partido Justicialista, ya que el Gobierno de Menem fue una marcada política neoliberal.

En Uruguay, el Frente Amplio en cabeza de Tabaré Vázquez y hoy con José Alberto Mujica lograrían igualmente llegar al más alto cargo de la Rama Ejecutiva. Desde 2008, en Paraguay, la Alianza Patriótica para el cambio con Fernando Lugo ganaría las elecciones presidenciales. Venezuela, desde la elección de Hugo Chávez, con el Movimiento v República, en cabeza del Partido Socialista Unido de Venezuela (psuv), tuvo su tercera reelección y posteriormente llegó a la Presidencia con Nicolás Maduro.

En Bolivia, desde el 2006, el Movimiento al Socialismo (MAS) con Evo Morales Ayma; en Brasil, con decepción similar a la que generó Menem en Argentina, Fernando Henrique Cardoso, un académico proveniente de la Teoría de la Dependencia que habría de impulsar un gobierno neoliberal, sólo con el Partido de los Trabajadores, desde el 2003, inicialmente con los dos periodos de Luiz Inácio Lula da Silva y ahora con Dilma Rousseff, sin que aún sea clara su tendencia. Ecuador en la figura de Rafael Correa realizó desde el 2007 con el movimiento Alianza País el giro hacia este mismo tipo de izquierda.

En Centroamérica, además de la nueva presidencia en Nicaragua de Daniel Ortega del Frente Sandinista de Liberación Nacional, y la presidencia en El Salvador desde 2009 de Mauricio Funes del Frente Farabundo Martí, cobran fuerza la Unidad Revolucionaria Nacional Guatemalteca en Guatemala, y han tenido notable importancia los gobiernos de Manuel Zelaya en Honduras, que tras provenir del liberalismo realizó un drástico cambio a favor de la nueva izquierda hasta que fue depuesto.

En México, el fenómeno Andrés Manuel López Obrador que con el Partido de la Revolución Democrática generó una importante discusión en torno a su

15. Véase Teodoro Petkoff. Las dos izquierdas. Revista Nueva Sociedad 197. Mayo-junio de 2005. candidatura presidencial, perdiendo en los comicios del primero de julio del 2012 con el electo presidente Enrique Peña Nieto. La elección de Ollanta Humala en el Perú permite prever un cambio en las políticas de gobierno en esa república que desde la reiterada presidencia de Alberto Fujimori, hoy condenado por graves violaciones a los derechos humanos, era en política regional un claro aliado de la política norteamericana a pesar de la procedencia partidista del presidente Alan García.

El futuro de este movimiento es hoy objeto de enconado enfrentamiento teórico, máxime si se tiene en cuenta que desde la propia derecha latinoamericana y el imperialismo, se han empeñado en generar una notable diferenciación entre casos como los de Brasil, Chile o Uruguay, a quienes catalogan como una izquierda "madura y responsable", y en el otro lado, se empeñan en colocar a regímenes como el cubano, el venezolano y ahora el boliviano y el ecuatoriano, estigmatizándolos como modelos "autoritarios, demagógicos y populistas".

El giro a la izquierda de sectores importantes de América Latina no significa necesariamente un peligro para la seguridad de Washington, el funcionamiento normal de los mercados y la seguridad jurídica de las empresas extranjeras en la región. El sueño del desarrollo capitalista, a pesar de lo probadamente irrealizable con que se reconquistó el tercer mundo, aún sigue conquistando los planes de una parte importante de esa "Nueva Izquierda". ${ }^{6}$

Claro que los cambios nos son profundos ni en lo económico, ni en lo político, ni en lo social, como sucediera en la Revolución mexicana o cubana. Debe entenderse que son cambios desde la reforma y no desde la revolución, y ello hace impensable, por lo menos en corto tiempo, una acelerada transformación de los problemas que han afectado durante siglos las inequitativas estructuras que mantienen la pobreza y la desigualdad. Son cambios muchas veces lentos y en ocasiones imperceptibles, que dependen en cada caso de la línea política que esté al mando, así como de la capacidad de encontrar el apoyo de la población. Parafraseando a Wallerstein, América Latina se ha movido de donde estaba hacia la izquierda. La continuidad del movimiento depende tanto de la geopolítica mundial, como del grado de cohesión y la lucidez de los programas de los movimientos sociales de izquierda. ${ }^{17}$

16. Atilio Borón. El mito del desarrollo capitalista nacional en la nueva coyuntura política de América Latina. ENSAYos CRíticos 3. 2007.

17. Immanuel Wallerstein. ¿Cómo se ha movido América Latina hacia la izquierda? La CRISIS estructural del Capitalismo. Ediciones Desde Abajo. (2007). 


\section{LA DINÁMICA ELECTORAL DE LOS GOBIERNOS ALTERNATIVOS}

Se esperaría que países que proponen un Estado y sociedad alternativos, además recurran a otros mecanismos distintos a la sanción para promover la participación política de la ciudadanía, como por ejemplo el debate y la alfabetización política. No obstante, haciendo la relación entre los denominados gobiernos alternativos y el tipo de sufragio que estos tienen, consideramos que sólo dos de estos países tienen un voto totalmente libre, por lo menos en cuanto a sus regulaciones constitucionales: Venezuela y Nicaragua.

$\mathrm{Al}$ ser una característica común a los gobiernos latinoamericanos que hacen parte de esta corriente política el ser todos de regímenes presidencialistas, veremos entonces cómo se ha presentado el fenómeno de la abstención electoral en los casos de Venezuela y Nicaragua para elecciones presidenciales.

En el caso de Nicaragua, existe la inscripción en el registro electoral como condición para poder ejercer el derecho al voto. Sin embargo, esta inscripción requiere solamente los requisitos de orden general (nacionalidad, edad), y desde luego la voluntad para estar en el señalado registro. Por lo tanto, quienes no se inscriben los asumimos como parte de la cifra de abstención. Veamos entonces en la tabla 1 los datos de la abstención electoral en Nicaragua en elecciones presidenciales, desde las siguientes elecciones en que triunfó el Frente Sandinista para la Liberación Nacional (FSLN), tras la guerra civil en 1979.

Tabla 1. Abstención electoral en elecciones presidenciales de Nicaragua después de 1979

\begin{tabular}{|l|c|c|c|c|c|}
\hline $\begin{array}{c}\text { FECHA } \\
\text { (Año) }\end{array}$ & POBLACIÓN TOTAL & $\begin{array}{c}\text { POBLACIÓN EN EDAD } \\
\text { DE VOTAR }\end{array}$ & $\begin{array}{c}\text { POBLACIÓN } \\
\text { QUE VOTó }\end{array}$ & \% VOTANTES & $\begin{array}{c}\text { \% DE ABSTENCIÓN } \\
\text { ELECTORAL }\end{array}$ \\
\hline $1984 \mathrm{a}$ & 3.162 .000 & 1.581 .000 & 1.170 .142 & $74,0 \%$ & $26,0 \%$ \\
\hline $1990 \mathrm{~b}$ & 3.871 .000 & 1.935 .500 & 1.510 .838 & $78,1 \%$ & $219 \%$ \\
\hline $1996 \mathrm{c}$ & 4.706 .000 & 2.447 .120 & 1.849 .362 & $75,6 \%$ & $24,4 \%$ \\
\hline $2001 \mathrm{~d}$ & 4.952 .226 & 2.827 .893 & 2.122 .233 & $75,0 \%$ & $25 \%$ \\
\hline $2006 \mathrm{e}$ & 5.570 .129 & 3.026 .097 & 2.244 .215 & $74,2 \%$ & $25,8 \%$ \\
\hline $2011 \mathrm{f}$ & 5.666 .301 & 3.637 .298 & 2.613 .087 & $71,8 \%$ & $28,2 \%$ \\
\hline
\end{tabular}

Fuente: información tomada del Institute for Democracy and Electoral Assistance. Disponible en: http://www.idea.int/

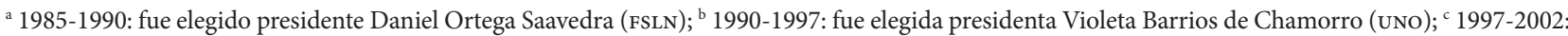

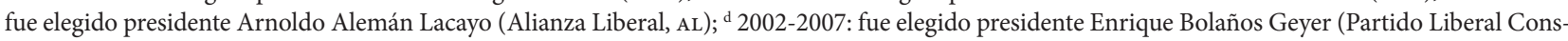
titucionalista); ${ }^{\mathrm{e}}$ 2007-2012: fue elegido presidente Daniel Ortega Saavedra. (FSLN); ${ }^{\mathrm{f}}$ 2012-2017: fue elegido presidente Daniel Ortega Saavedra. (FSLN)

Lo anterior nos muestra un panorama de abstención relativamente estable que oscila en las últimas seis elecciones presidenciales, variable entre el $21,9 \%$ y el $28,2 \%$ de abstención. La abstención de las elecciones de 2011 fue la más alta en las últimas elecciones, o por lo menos desde que participa el FSLN.

Teniendo en cuenta un periodo similar, el caso venezolano no es tan sosegado como el de Nicaragua si nos referimos a la abstención electoral. ${ }^{18}$ Como veremos en la tabla 2, en el periodo que va desde 1983 hasta 2013 encontramos cifras que oscilan entre el 12,25\% de abstención y el 43,69\%.

18. Para una interpretación más amplia sobre la abstención electoral en Venezuela, puede verse nuestro artículo: Abstención electoral: una mirada al caso venezolano.
Tabla 2. Abstención electoral en Venezuela entre 1983 y 2013

\begin{tabular}{|c|c|}
\hline AÑO & $\begin{array}{c}\text { \% ABSTENCIÓN } \\
\text { ELECTORAL }\end{array}$ \\
\hline 1983 & 12,25 \\
\hline 1988 & 18,1 \\
\hline 1993 & 39,84 \\
\hline 1998 & 36,54 \\
\hline 2000 & 43,69 \\
\hline 2006 & 25,3 \\
\hline 2012 & 19,51 \\
\hline 2013 & 20,22 \\
\hline
\end{tabular}

Fuente: datos tomados de la Dirección de Estadísticas Electorales del Consejo Nacional Electoral de Venezuela. Disponible en http://www. cne.gob.ve 
Hasta 1988, muestra estabilidad el proceso electoral venezolano con la elección de Carlos Andrés Pérez. La crisis de finales de los años ochenta del siglo pasado por la aplicación de las políticas macroeconómicas neoliberales, afectó de forma sentida la legitimidad del régimen político venezolano, desbordando en el denominado caracazo en 1989, como se evidencia en la tabla 2.

Es marcado el desencanto de los venezolanos comenzando la década de los años noventa y esto se reflejará en la alta abstención de 1993, modificándose sólo levemente la cifra en las elecciones de 1998. Sin embargo, vuelve a sentirse una alta abstención en el 2000, explicable si se tiene en cuenta que estas elecciones generales se realizaron apenas un año y medio después de la elección presidencial, y que los partidos tradicionales del puntofijismo Alianza Democrática y COPEI no se presentaron a los comicios buscando deslegitimar al Gobierno en las urnas.

Las elecciones presidenciales que se realizaron en Venezuela en el 2006 correspondieron al periodo ejecutivo 2007-2013. A pesar de que el Consejo Nacional Electoral admitió veintitrés candidatos presidenciales, los resultados mostraron la polarización que desde hace algunos lustros vive el país entre el entonces candidato Hugo Chávez (Q. E. P. D.) y la oposición, representada especialmente por los sectores que conformaron el puntofijismo, Acción Democrática y COPEI. Las elecciones arrojaron un resultado favorable para el candidato del Movimiento v República y sus sectores de coalición con un $62,84 \%$ de los votos, seguido del candidato Manuel Rosales con el 36,9\%. Los otros doce candidatos que se presentaron tuvieron todos menos del $1 \%$ de los votos, mostrando los resultados una notable concentración de la votación en los dos primeros.

Las últimas elecciones presidenciales regulares de Venezuela fueron el 7 de octubre de 2012, oportunidad para confirmar una tendencia que no se trataba de lo ocurrido en el 2006: un simple efecto coyuntural de las políticas del gobierno del presidente Hugo Chávez Frías.

Finalmente, el 14 de abril de 2013, tras la muerte del presidente Chávez, ${ }^{19}$ se realizaron elecciones presidenciales atípicas que llevaron a la Presidencia al hoy mandatario Nicolás Maduro con un 50,61\% de los votos, confirmando una tendencia participativa en las

19. Al parecer, se trató de un asesinato, según lo sostienen voces políticas y académicas como la del doctor Atilio Borón, ex secretario ejecutivo del Consejo Latinoamericano de Ciencias Sociales cLAcso. Véase http://actualidad.rt.com/actualidad/view/103501-muerte-hugo-chavezmagnicidio-cia elecciones venezolanas tras la llegada del chavismo al gobierno, y en las cuales sólo aumentó la abstención en menos de un punto, a pesar del ambiente de frustración de las mayorías por la muerte del caudillo popular.

\section{CONCLUSIONES}

De todo lo anterior, podemos concluir que, tomando en cuenta exclusivamente los casos de Nicaragua y Venezuela (los dos únicos países de tendencias alternativas que no tienen voto obligatorio), los cambios o resultados no han sido idénticos.

Mientras que en Nicaragua la llegada del gobierno del FSLN no ha significado cambios importantes en la cifra de abstención electoral (oscilando entre el 21 y el 28\%), para el caso de Venezuela hay importantes cambios cuantitativos que permiten inferir la influencia del gobierno alternativo en la dinámica electoral, reduciéndose enormemente la cifra de abstención tras la crisis que se viviera desde comienzos de los años noventa del siglo pasado.

Claro está que no merece desprecio el ambiente de polarización que se ha vivido en Venezuela desde hace un poco más de una década, y que bien puede signarse por dos hechos que marcaron una inestabilidad institucional: el caracazo de 1989, y algunos años después, el intento de golpe de Estado en el 2002.

\section{REFERENCIAS}

Alejo Vargas. Viejas y nuevas izquierdas y los gobiernos alternativos en América Latina. Coord. Jairo Estrada. MARX VIVE: IZQUIERDA Y SOCIALISMO EN AMÉRICA LATINA. (2008). Págs. 121-131.

Atilio Borón. El mito del desarrollo capitalista nacional en la nueva coyuntura política de América Latina. Ensayos CRÍTICOS 3. 2007.

Beatriz Stolowicz. Gobiernos de IZQUierda eN AméricA Latina. Ediciones Aurora. (2008).

Ciska Raventós. Abstención: CONCEPTOS Y ALCANCES, PROBLEMAS ASOCIADOS Y POSIBILIDADES DE SUPERACIÓN. Perspectiva política. Xvil Conferencia Protocolo de Tikal. Centro Interamericano de Asesoría y Promoción Electoral (CAPEL). (2003).

Immanuel Wallerstein. ¿Cómo se ha movido América Latina hacia la izquierda? LA CRISIS ESTRUCTURAL DEL CAPITAlismo. Ediciones Desde Abajo. (2007). 
Instituto Interamericano de Derechos Humanos y Centro Interamericano de Asesoría y Promoción Electoral [IIDH y CApel]. Diccionario electoral. Tomo I. (2000).

José Thompson. Abstencionismo y participación electoral. TRatado De DeRecho electoral comparado De América Latina. Fondo de Cultura Económica. (2007). Págs. 266-286.

Juan Hernández. Abstención activa. DiCCIONARIO CRÍTICO DE CiENCIAS SOCIALES. Universidad de La Laguna. (2004).

Mark Moisevich Rosental y Pavel Fedorovich Iudin. DicCIONARIO SOVIÉTICo DE FILOSOFÍA. Ediciones Pueblos Unidos. (1965).
Manuel Aragón. Derecho de sufragio: principio y función. TRATADO DE DERECHO ELECTORAL COMPARADO DE AMÉRICa Latina. Fondo de Cultura Económica. (2007).

Max Weber. Economía y sociedad. Fondo de Cultura Económica. (1997).

Nicolás Maquiavelo. El Príncipe. Editorial Porrúa. (1994).

Pierre Rosanvallon. La LEGITIMIDAD DEMOCRÁtica. IMPARCIALIDAD, REFLEXIVIDAD Y PROXIMIDAD. Ediciones Paidós Ibérica. (2010).

Teodoro Petkoff. Las dos izquierdas. Revista NUeva socieDAD 197. Mayo-junio de 2005. 